\title{
Efecto de la disponibilidad de agua sobre una gramínea invasora del caldenal
}

\author{
Ruth B. Rauber ${ }^{12, \pm}$; Manuel R. Demaría ${ }^{1}$ \& Diego F. Steinaker ${ }^{3}$ \\ ${ }^{1}$ INTA EEA San Luis. ${ }^{2}$ Consejo Nacional de Investigaciones Científicas y Técnicas (CONICET). ${ }^{3}$ University of Regina, \\ Canadá.
}

\begin{abstract}
Resumen. En los últimos años, en la región semiárida central argentina se observó el incremento de especies gramíneas nativas no palatables $C_{3}$ conocidas generalmente como "pajas". Una causa posible del éxito de estas especies es el aumento de las precipitaciones observado en la región en los últimos años. El objetivo de este trabajo fue evaluar el efecto de una mayor disponibilidad de agua y su distribución estacional sobre la biomasa y la producción de estructuras reproductivas de una especie $\mathrm{C}_{3}$ no palatable y altamente invasiva (Jarava ichu), y una especie $\mathrm{C}_{4}$ no palatable (Setaria lachnea). Para ello se realizó un ensayo en invernáculo que consistió en riego manual de individuos de ambas especies en macetas. Los tratamientos fueron: año seco, año húmedo con pico otoñal y año húmedo con pico primaveral. El aumento de la disponibilidad de agua favoreció la biomasa de hojas y tallos de J. ichu independientemente de la distribución estacional, con respecto al año seco. Por el contrario, la biomasa de $S$. lachnea no respondió a los distintos tratamientos $(P>0.05)$. Asimismo, el número de panojas fue mayor para J. ichu bajo el tratamiento año húmedo con pico primaveral, pero no se detectaron diferencias para S. lachnea.
\end{abstract}

[Palabras clave: invasión, fenología, precipitaciones]

\begin{abstract}
AвstRACt. Effect of the availability of water on an invasive grass of the Caldenal Region. In the last years, in the semi-arid central region of Argentina, the increase of C3 non-palatable native grass species known generally as "pajas" was observed. One possible cause of the success of these species is the increase in rainfall observed in the region in recent years. The objective of this work was to evaluate the effect of a greater water availability and its seasonal distribution on the biomass and the production of reproductive structures of a non-palatable and highly invasive $\mathrm{C}_{3}$ species (Jarava ich $u$ ) and a non-palatable $\mathrm{C}_{4}$ species (Setaria lachnea). For this, a greenhouse experiment was carried out, consisting in manual irrigation of individuals of both species in pots. Treatments were: dry year, wet year with autumnal peak and wet year with spring peak. The increase in water availability favored the biomass of leaves and stems of $J$. ich $u$, independently of the seasonal distribution, with respect to the dry year. On the contrary, the biomass of $S$. lachnea did not respond to the different treatments $(P>0.05)$. Likewise, the number of panicles was greater for $J$. ich $u$ under the wet year treatment with spring peak, but there were no differences for S. lachnea.
\end{abstract}

[Keywords: invasion, phenology, precipitations]

\section{INTRODUCCIÓN}

Las comunidades vegetales pueden verse impactadas por factores antrópicos y climáticos (Milchunas and Lauenroth 1993). Ante determinados disturbios, algunas especies resultan beneficiadas y aumentan su abundancia con respecto a las demás (Fleischner 1994; MacLeod and McIvor 2006; Rogstad et al. 2009; Vavra et al. 2007); incluso, suelen alcanzar coberturas tan altas que causan pérdida y fragmentación de hábitats o disminución de la capacidad productiva (Eviner et al. 2010; MacLeod and McIvor 2006; Milton and Dean 1995).

Los bosques de caldén de la región semiárida central argentina están amenazados por la continua incorporación de tierras a la agricultura (Demaría et al. 2008; Maceira

Editora asociada: Marina Omacini et al. 1996) y por la degradación florística y productiva de los bosques remanentes usados con fines ganaderos (Fernández et al. 2009; Gabutti et al. 2009; Menéndez and La Rocca 2006; Morici et al. 2009). Una de las consecuencias de esta degradación es el aumento de especies gramíneas nativas no palatables $\mathrm{C}_{3}$, conocidas generalmente como "pajas" (Jarava ichu Ruiz and Pav., antes Stipa eriostachya Kunth, y Nassella tenuissima (Trin.) Barkworth, antes Stipa tenuissima Trin). El pastoreo intenso fue señalado como la causa principal del aumento de cobertura de estas especies debido a la sobrecarga ganadera aplicada sobre estos pastizales (Fernández et al. 2009). Sin embargo, estudios recientes mostraron que la mayor abundancia de pajas estaría relacionada con intensidades intermedias de pastoreo (Rauber et al. 
2014). Además, las especies $C_{4}$ no palatables también deberían haber aumentado su rango de distribución en respuesta al pastoreo, pero esta situación no ha sido registrada de manera extensiva en los bosques de caldén. Por lo tanto, es posible que otros factores no evaluados (e.g., el incremento significativo de las precipitaciones registrado a partir de la segunda mitad del siglo XX [Bertón et al. 2006; Viglizzo et al. 1997] y su desplazamiento de la primavera hacia el otoño) podrían ser factores adicionales causantes de la dominancia de especies $C_{3}$ no palatables, en detrimento de especies $C_{4}^{3}$.

Las pajas se podrían ver beneficiadas con el aumento de las precipitaciones, ya que las especies $C_{3}$, en condiciones de exceso de agua, tendrían un gasto energético menor que las $C_{4}$ (Shuli et al. 2005). Las especies $C_{4}$ son dominantes en ambientes abiertos, cálidos y áridos (Osmond et al. 1982), y tienen una mejor adaptación al estrés hídrico y una mayor eficiencia en el uso del agua (Nayyar and Gupta 2006). Ambos grupos funcionales presentan inhibición de la fotosíntesis en situaciones de estrés hídrico (Ghannoum 2009; Nayyar and Gupta 2006). Por otra parte, fue demostrado que las precipitaciones y su distribución estacional son controles clave de la distribución de la abundancia relativa de las especies $\mathrm{C}_{3}$ y C $_{4}$ (Hattersley 1983; Paruelo et al. 1999; Paruelo and Lauenroth 1996). En algunos sistemas se observó que las especies $\mathrm{C}_{4}$ son más numerosas en veranos cálidos y húmedos, y las C3 en primaveras frescas y húmedas (Hattersley 1983). Las pajas tienen un ciclo de crecimiento otoño-invierno-primaveral (de marzo a octubre), con producción de inflorescencias en primavera (de octubre a diciembre). En cambio, las gramíneas $\mathrm{C}_{4}$ tienen un ciclo de crecimiento primavero-estival (de septiembre a diciembre), con producción de inflorescencias en verano (de enero a marzo). En la región semiárida, los otoños húmedos podrían favorecer la producción de biomasa y la capacidad de generar estructuras reproductivas en el comienzo de la estación de crecimiento de las especies $\mathrm{C}_{3}$, con respecto a las $\mathrm{C}_{4}$ o viceversa, favoreciendo a las especies de cada tipo funcional por una mayor disponibilidad de agua al final de la estación de crecimiento. El primer patrón coincidiría con una mayor actividad fotosintética observada en especies $C_{3}$ en otoños húmedos, comparado con primaveras húmedas (Niu et al. 2005), y el segundo patrón coincidiría con el observado por Hattersley (1983).
A pesar de la gran importancia ecológica y agronómica relacionada con la invasión de pajas en la región semiárida central argentina, no existen estudios experimentales tendientes a evaluar los posibles factores implicados (Rauber et al. 2014). El objetivo de este trabajo fue evaluar el efecto de una mayor disponibilidad de agua y su distribución estacional sobre la biomasa y la producción de estructuras reproductivas de una especie $\mathrm{C}_{3}$ no palatable y altamente invasiva (Jarava $i c h u$ ), y una especie $C_{4}$ no palatable (Setaria lachnea). Ambas son especies perennes, de reproducción exclusiva por producción de semillas, y en la región se las considera claves por su dominancia, por los efectos sobre la disponibilidad de forraje y como indicadoras de la condición del pastizal (Kunst et al. 2007).

\section{MATERIALES Y MÉTOdOS}

Se realizó un experimento manipulativo en tres invernáculos ubicados en la Estación Experimental San Luis del INTA (Instituto Nacional de Tecnología Agropecuaria) en Villa Mercedes (San Luis, Argentina). En octubre de 2011, en cada invernáculo se establecieron 42 macetas (126 macetas en total). A cada una de ellas se trasplantó, al azar, un individuo adulto de J. ichu o $S$. lachnea en partes iguales (21 individuos de cada especie en cada invernáculo, para un total de 63 individuos por especie). Cada uno de los tres invernáculos representó un bloque o repetición. Los invernáculos (6 m de largo x 3.8 $\mathrm{m}$ de ancho $\mathrm{x} 1.5 \mathrm{~m}$ de alto) fueron construidos con paredes y techo de plástico transparente (Agropol cristal) de $200 \mu \mathrm{m}$ para evitar las precipitaciones y diferencias de calidad de luz entre tratamientos. Las macetas consistieron en grandes tambores plásticos de $0.5 \mathrm{~m}$ de diámetro por $0.8 \mathrm{~m}$ de profundidad $\left(0.157 \mathrm{~m}^{3}\right)$ enterrados en el suelo y con fondo abierto para que el agua drenara libremente. Estas macetas evitan la competencia radical entre plantas con distintos tratamientos y aseguran un mejor control sobre las condiciones de riego de cada tambor. A las macetas se las llenó con suelo representativo del área de estudio (Ustortente típicos, textura franco-arenosa). Durante la primera estación de crecimiento se regó con suficiente agua para lograr que las plantas se establecieran correctamente.

Para la selección de los tratamientos se consideraron las características pluviométricas de la región. Se tomaron los datos de 

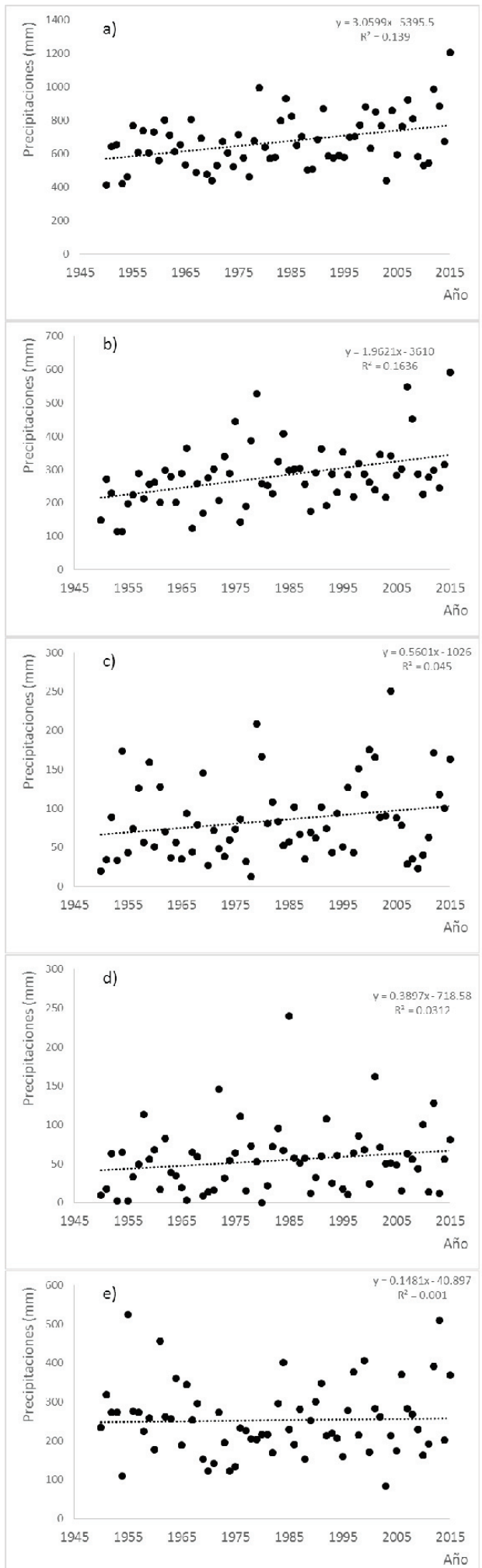

Figura 1. Cambios en las precipitaciones para el período 1960-2015. a) Total $(P=0.002)$, b) verano $(P<0.001)$, c) otoño $(P=0.087), \mathrm{d})$ invierno $(P=0.156)$, e) primavera $(P=0.804)$.

Figure 1. Changes in rainfall for the period 1960-2015. a) Total $(\mathrm{p}=0.002), \mathrm{b})$ summer $(\mathrm{p}<0.001), \mathrm{c})$ autumn $(\mathrm{p}=$ $0.087), d)$ winter $(p=0.156)$, e) spring $(p=0.804)$. precipitaciones mensuales de la estación meteorológica de la estación experimental de INTA San Luis durante el período 19502010. Con estos datos se realizó un análisis de regresión para evaluar las tendencias a lo largo del tiempo. Se observó un aumento de las precipitaciones (Figura 1a) concentrado en las estaciones de verano y de otoño (Figura 1 be). Por otra parte, se observó una disminución de las precipitaciones en primavera (Figura 1e). Para evaluar el efecto de la disponibilidad de agua y de su distribución estacional en la producción de biomasa y de órganos reproductivos en especies con distintas rutas metabólicas, elegimos representar tres tratamientos. Los tratamientos fueron: año seco (358 mm/año de precipitación), año húmedo (864 mm/año) con pico otoñal y año húmedo (864 mm/año) con pico primaveral (Figura 2). Estos valores representan el primer y tercer cuartil por mes de la serie histórica de precipitaciones. Estos regímenes de riego fueron distribuidos al azar a las plantas de cada especie y en cada invernáculo con aplicaciones de hasta dos riegos manuales semanales durante 2 años (marzo de 2012 a marzo de 2014) a siete individuos de cada especie en cada invernáculo.

Al final del tratamiento se determinó para cada maceta, mediante cortes, la biomasa aérea total (hojas y tallos, incluyendo el material senescente), el número de panojas reproductivas (con láminas y tallos, medidas a partir del material cosechado) y el peso seco total por maceta. El material recolectado se secó en estufa a $60{ }^{\circ} \mathrm{C}$ durante $48 \mathrm{~h}$, hasta peso constante y luego se pesó. Decidimos medir en ambas especies las variables respuesta en marzo de 2014 para garantizar el mismo periodo de tiempo de aplicación de

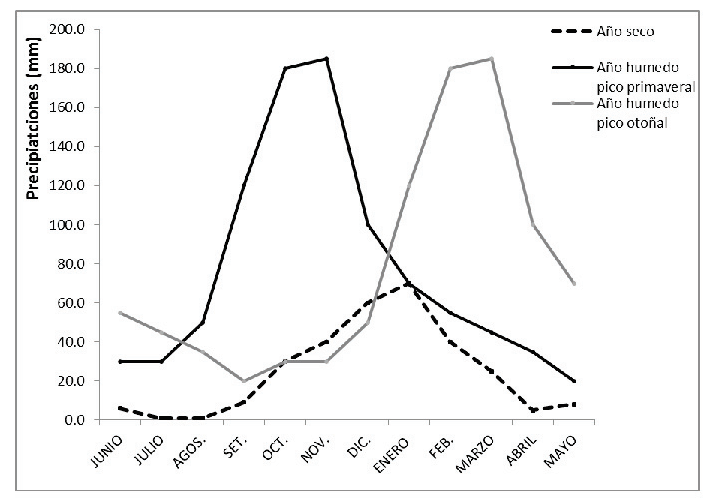

Figura 2. Distribución de los regímenes de disponibilidad de agua.

Figure 2. Distribution of water availability regimes. 
los tratamientos de riego en las dos especies estudiadas. De esta manera priorizamos la aplicación de tratamientos idénticos en ambas especies, con idénticos periodos de tiempo de crecimiento acumulado para cada una.

La biomasa aérea total, el número de panojas reproductivas y el peso seco de las panojas para cada especie se compararon contra el tratamiento de riego mediante una prueba LSD de Fisher de análisis de varianza con diseño en bloques. Se consideró cada invernáculo como un bloque y se tomó como covariable el diámetro de corona inicial de cada planta. Para los análisis se utilizó el software InfoStat v2016 (Di Rienzo et al. 2016).

\section{Resultados y Discusión}

El aumento de la disponibilidad de agua favoreció el desarrollo de biomasa de hojas y tallos de J. ichu, independientemente de la distribución estacional de las lluvias, con respecto al año seco (Tabla 1). Por el contrario, la biomasa de $S$. lachnea no respondió a los distintos tratamientos $(P>0.05)$. El número de panojas reproductivas fue mayor para $J$. ichu bajo el tratamiento año húmedo con pico primaveral y no se observaron diferencias en S. lachnea bajo ningún tratamiento (Tabla 1). Por último, no hubo diferencias entre tratamientos cuando se evaluó el peso de las panojas, para ninguna de las dos especies. Por lo tanto, el aumento de biomasa reproductiva en J. ichu está principalmente relacionado con un incremento en el número de panojas y no con su tamaño.

Si bien el cambio en la distribución estacional de las precipitaciones observado a nivel regional no sería un factor que estaría

Tabla 1. Biomasa final, número de panojas y peso de panojas reproductivas para cada especie y tratamiento ( $\mathrm{S}=$ año seco; $\mathrm{HO}=$ año húmedo con pico otoñal; $\mathrm{HP}=$ año húmedo con pico primaveral) al finalizar el segundo año de tratamiento. Letras diferentes indican diferencias entre tratamientos (LSD Fisher, $P<0.05$ ).

Table 1. Final biomass, number of panicles and total weight of panicles for each species and treatment $(S=$ dry year, $\mathrm{HO}=$ wet year with autumnal peak, $\mathrm{HP}=$ wet year with spring peak) after two years of treatment. The different letters indicate differences between treatments (LSD Fisher, $\mathrm{p}<0.05$ ).

\begin{tabular}{llccc}
\hline & & $\mathrm{S}$ & $\mathrm{HO}$ & $\mathrm{HP}$ \\
\hline \multirow{2}{*}{ Biomasa (g) } & J.ichu & $59,03 \mathrm{a}$ & $113,08 \mathrm{~b}$ & $104,46 \mathrm{~b}$ \\
& S.lachnea & $45,74 \mathrm{a}$ & $47,66 \mathrm{a}$ & $44,43 \mathrm{a}$ \\
\multirow{2}{*}{ Nro panojas } & J.ichu & $106,44 \mathrm{a}$ & $131,52 \mathrm{a}$ & $180,42 \mathrm{~b}$ \\
& S.lachnea & $21,66 \mathrm{a}$ & $24,87 \mathrm{a}$ & $21,98 \mathrm{a}$ \\
\multirow{2}{*}{ Peso panojas (g) } & J.ichu & $6,63 \mathrm{a}$ & $6,22 \mathrm{a}$ & $7,63 \mathrm{a}$ \\
& S.lachnea & $3,27 \mathrm{a}$ & $4,11 \mathrm{a}$ & $3,25 \mathrm{a}$ \\
\hline
\end{tabular}

condicionando un aumento en la producción de estructuras reproductivas y vegetativas, los resultados obtenidos en este trabajo indican que un aumento en la disponibilidad de agua favorecería a la especie invasora $J$. $i c h u$, y probablemente a otras especies $\mathrm{C}_{3^{\prime}}$ en detrimento de especies $C_{4}$. Las especies $\mathrm{C}_{4}$ tendrían menores tasas de crecimiento de biomasa y de panojas reproductivas, y las plantas $C_{3}$ serían favorecidas en condiciones de alta disponibilidad hídrica debido a que la fijación de carbono requiere menor gasto energético en estas especies (Shuli et al. 2005). Por otro lado, en situación de coexistencia de ambas especies en sistemas naturales, las especies $\mathrm{C}_{3}$ poseerían ventaja competitiva sobre las $\mathrm{C}_{4}$ a través de una mayor producción de semillas en años con primaveras húmedas (Pearcy et al. 1984).

La falta de respuesta de $S$. lachnea a los tratamientos no concuerda con lo observado por otros autores en condiciones de laboratorio para especies $C_{4}$ (Nayyar and Gupta 2006; Ripley et al. 2007). En estos estudios se observaron aumentos en la producción de biomasa vegetativa ante un incremento de las precipitaciones. Asimismo, a escala regional se determinó mediante ensayos biogeográficos que la abundancia de especies $\mathrm{C}_{4}$ disminuye a medida que las precipitaciones decrecen, debido a una reducción en la eficiencia fotosintética en condiciones de sequía severa (Ripley et al. 2007). Es posible que la especie que se utilizó en este ensayo alcance su óptimo fisiológico aun cuando la disponibilidad de agua sea muy baja (358 mm de precipitación anual). Por lo tanto, bajo las condiciones experimentales de este ensayo, el agua no habría sido un factor limitante para S. lachnea en ninguno de los 3 tratamientos y su crecimiento habría estado limitado por otros factores intrínsecos o extrínsecos (e.g., disponibilidad de luz, nutrientes, etc.).

Como conclusión, el aumento en la disponibilidad de agua tendría un efecto clave en la expansión de J. ichu en la región. En coincidencia con estudios previos (Hattersley 1983), la especie $C_{3}$ resultó favorecida por una mayor disponibilidad total de agua y por las primaveras húmedas para incrementar el potencial reproductivo en las condiciones creadas durante el experimento. El efecto combinado de los incrementos, tanto en la presión de pastoreo (Rauber et al. 2014) como en las precipitaciones, podría ser el factor determinante de la invasión de pajas en el semiárido central argentino. 
Agradecimientos. A Karina Frigerio, Marcelo Scibilia y Aldo Suárez por la ayuda brindada. Trabajo financiado por los proyectos regionales de INTA La Pampa - San Luis (PAMSL-1282206), y por una beca posdoctoral de CONICET a R. B. Rauber.

\section{REFERENCIAS}

Bertón, J. A., J. C. Echeverría, and E. G. Jobbágy. 2006. Cambio Climático. En: J. C. Echeverría, E. G. Jobbágy and A. D. Collado (eds.). Aptitud forestal de la provincia de San Luis. Editorial Payne. San Luis. Argentina. Pp. 230.

Demaría, M. R., I. Aguado Suárez, and D. F. Steinaker. 2008. Reemplazo y fragmentación de pastizales pampeanos semiáridos en San Luis, Argentina. Ecología Austral 18:55-70.

Di Rienzo, J. A., F. Casanoves, M. G. Balzarini, L. González, M. Tablada, and C. W. Robledo. InfoStat versión 2016. Grupo InfoStat, FCA, Universidad Nacional de Córdoba, Argentina. URL: www.infostat.com.ar.

Eviner, V. T., S. A. Hoskinson, and C. V. Hawkes. 2010. Ecosystem impacts of exotic plants can feed back to increase invasion in western US rangelands. Rangelands 32:21-31.

Fernández, O. A., M. E. Gil, and R. A. Distel. 2009. The challenge of rangeland degradation in a temperate semiarid region of Argentina: The Caldenal. Land Degradation and Development 20:431-440.

Fleischner, T. L. 1994. Ecological costs of livestock grazing in Western North America. Conservation Biology 8:629644

Gabutti, E. G., N. O. Maceira, V. Gómez Hermida, and J. L. Leporati. 1 2009. Caracterización de la diversidad florística en el caldenal. Provincia Fitogeográfica del Espinal. San Luis. Tesis de Magister. Universidad Nacional de San Luis.

Ghannoum, O. 2009. C4 photosynthesis and water stress. Annals of Botany 103:635-644.

Hattersley, P. W. 1983. The distribution of C3 and C4 grasses in Australia in relation to climate. Oecologia 57:113128.

Kunst, C., R. Ledesma, E. Monti, J. Casillo, and J. Godoy. 2007. Gramíneas indicadoras de condición en sitios de pastizal del sudoeste de Santiago del Estero. RIA 36:33-61.

Maceira, N. O., C. Saravia Toledo, C. Bertonatti, and A. Vila. 1996. Conservación y uso sostenible de la biodiversidad en las zonas áridas y semiáridas de Argentina. Pp. 13-48 en J. Simonetti (ed.). Conservación y uso sostenible de la biodiversidad en zonas áridas y semiáridas de América Latina y El Caribe. Oficina Regional de la FAO para América Latina y El Caribe, Serie Zonas Áridas y Semiáridas 8.

MacLeod, N. D., and J. G. McIvor. 2006. Reconciling economic and ecological conflicts for sustained management of grazing lands. Ecological Economics 56:386-401.

Menéndez, J. L., and S. M. La Rocca. 2006. Primer inventario nacional de bosques nativos. Informe Regional Espinal, Segunda Etapa. ANEXO I: Estado de Conservación del Distrito Caldén.

Milchunas, D. G., and W. K. Lauenroth. 1993. Quantitative Effects of Grazing on Vegetation and Soils over a Global Range of Environments. Ecological Monographs 63:327-366.

Milton, S. J., and W. R. J. Dean. 1995. South Africa's arid and semiarid rangelands, why are they changing and can they be restored? Environmental Monitoring and Assessment 37:245-264.

Morici, E., V. Doménech-García, G. Gómez-Castro, A. Kin, A. Saenz, and C. Rabotnikof. 2009. Diferencias estructurales entre parches de pastizal del Caldenal y su influencia sobre el banco de semillas, en la provincia de La Pampa, Argentina. Agrociencia 43:529-537.

Nayyar, H., and D. Gupta. 2006. Differential sensitivity of C3 and C4 plants to water deficit stress: Association with oxidative stress and antioxidants. Environmental and Experimental Botany 58:106-113.

Niu, S., Z. Yuan, Y. Zhang, W. Liu, L. Zhang, J. Huang, and S. Wan. 2005. Photosynthetic responses of C3 and C4 species to seasonal water variability and competition. Journal of Experimental Botany 56:2867-2876.

Osmond, C. B., K. Winter, and H. Ziegler. 1982. Functional significance of different pathways of CO2 fixation in photosynthesis. Pp. 479-547 in O. L. Lange, P. S. Noble, C. B. Osmond and H. Ziegler (eds.). Encyclopedia of Plant Physiology. New Series, Vol. 12B. Berlin: Springer Verlag.

Paruelo, J. M., and W. K Lauenroth. 1996. Relative abundance of plant functional types in grasslands and shrublands of North America. Ecological Applications 6:1212-1224.

Paruelo, J. M., W. K. Lauenroth, I. C. Burke, and O. E. Sala. 1999. Grassland precipitation-use efficiency varies across a resource gradient. Ecosystems 2: 64-68.

Pearcy, R. W., and J. Ehleringer. 1984. Comparative ecophysiology of C3 and C4 plants. Plant, Cell and Environment 7:1-13.

Rauber, R., D. Steinaker, M. Demaría, and D. Arroyo. 2014. Factores asociados a la invasión de pajas en bosques de la región semiárida central argentina. Ecología Austral 24:320-326.

Ripley, B. S., M. E. Gilbert, D. G. Ibrahim, and C. P. Osborne. 2007 Drought constraints on C4 photosynthesis: stomatal and metabolic limitations in C3 and C4 subspecies of Alloteropsis semialata. Journal of Experimental Botany 58:13511363.

Rogstad, A., T. M. Bean, A. Olsson, and G. M. Casady. 2009. Fire and invasive species management in hot deserts: resources, strategies, tactics, and response. Rangelands 31:6-13.

Shuli, N., Y. Zhiyou, Z. Yanfang, L. Weixeing, Z. Lei, H. Jianhui, and S. Wan. 2005. Photosynthetic responses of C3 and C4 species to seasonal water variability and competition. Journal of Experimental Botany 56:2867-2876.

Vavra, M., C. G. Parks, and M. J. Wisdom. 2007. Biodiversity, exotic plant species, and herbivory: The good, the bad, and the ungulate. Forest Ecology and Management 246:66-72.

Viglizzo, E. F., Z. E. Roberto, F. A. Lértora, E. López Gay, and J. Bernardos. 1997. Climate and land-use change in fieldcrop ecosystem of Argentina. Agric Ecosyst Environ 66:61-70. 